\title{
Espécies medicinais comercializadas como "quebra-pedras" em Porto Alegre, Rio Grande do Sul, Brasil
}

\author{
Adriana Morais Aita, ${ }^{1}$ Hélio Nitta Matsuura, ${ }^{1}$ Clarice Azevedo Machado, ${ }^{2}$ \\ Mara Rejane Ritter ${ }^{*}, 1$ \\ ${ }^{1}$ Departamento de Botânica, Instituto de Biociências, Universidade Federal do Rio Grande do Sul. Av. Bento \\ Gonçalves, 9500, 91509-900 Porto Alegre-RS, Brasil, \\ ${ }^{2}$ Faculdade de Farmácia, Pontifícia Universidade Católica do Rio Grande do Sul, Av. Ipiranga, 6681, 90619-900 \\ Porto Alegre-RS, Brasil
}

\begin{abstract}
RESUMO: Este trabalho visa identificar as espécies comercializadas como quebra-pedras em Porto Alegre, verificar indícios de toxicidade e estabelecer padrões morfológicos para diferenciação das mesmas. São espécies utilizadas na medicina popular principalmente para afecções nos rins e bexiga bem como diuréticas. As 16 amostras foram adquiridas de 14 comerciantes atuantes em bancas fixas ou em feiras-livres. Realizou-se uma revisão bibliográfica para levantamento de dados referentes à utilização de plantas medicinais com o nome de quebra-pedras em diferentes cidades do Rio Grande do Sul. Indícios de toxicidade, dados químicos e biológicos destas plantas foram revisados em diferentes bases de dados e em bibliografia específica. Identificaram-se sete espécies comercializadas como quebra-pedras em Porto Alegre: Euphorbia prostrata e E. serpens (Euphorbiaceae), Phyllanthus niruri e P. tenellus (Phyllanthaceae), Desmodium incanum (Fabaceae), Cunila microcephala (Lamiaceae) e Heimia salicifolia (Lythraceae). Destas, as espécies de Phyllanthus e Euphorbia são as que apresentam maior semelhança morfológica quando comercializadas secas e fragmentadas.
\end{abstract}

Unitermos: Quebra-pedras, medicina popular, morfologia.

\begin{abstract}
Medicinal species sold as "quebra-pedras" in Porto Alegre, Rio Grande do Sul, Brazil". The aims of this work were to identify the species sold as "quebra-pedras" in Porto Alegre, verify signs of toxicity and establish morphological patterns that help to distinguish such species. Those species are believed to be diuretic and are used in popular medicine mainly to treat kidney and bladder affections. The samples (16) were purchased from 14 sellers who work in permanent market stalls or in public marketplaces. A bibliographic revision gathered data on the use of medicinal plants called "quebra-pedras" in several cities of Rio Grande do Sul. Signs of toxicity, chemical and biological data of these plants were reviewed from different databases and specific bibliography. Seven species were identified among those sold as "quebra-pedras" in Porto Alegre: Euphorbia prostrata and E. serpens (Euphorbiaceae), Phyllanthus niruri and P. tenellus (Phyllanthaceae), Desmodium incanum (Fabaceae), Cunila microcephala (Lamiaceae) and Heimia salicifolia (Lythraceae). The greater morphological similarity when dried and fragmented plants are sold together was verified between Phyllanthus and Euphorbia.
\end{abstract}

Keywords: “Quebra-pedras”, popular medicine, morphology.

\section{INTRODUÇÃO}

Cada vez mais a população brasileira busca alternativas para o tratamento de doenças. A maioria das pessoas não busca atendimento médico, consumindo produtos que podem colocar em risco sua saúde.

Zuccolotto et al. (1999) verificaram a ocorrência de problemas na qualidade de produtos fitoterápicos comercializados em Porto Alegre, tais como substituição de farmacógenos, contaminações e ausência do constituinte químico principal. Ressaltam ainda que as amostras provenientes de ervateiros foram as que apresentaram o maior índice de rejeição da qualidade.
Acidentes com o uso de plantas podem ocorrer pela alta concentração de doses, pelo estado de conservação das plantas e a forma de uso. A identificação incorreta de plantas, bem como o uso de diferentes plantas com a mesma indicação ou o mesmo nome popular, pode levar a intoxicações (Rates, 2001).

Diferentes espécies conhecidas como quebrapedras são utilizadas popularmente para problemas renais no Rio Grande do Sul, muitas comercializadas fragmentadas, dificultando a identificação correta das espécies.

Neste trabalho pretendeu-se estabelecer diferenças morfológicas significativas entre as espécies comercializadas como quebra-pedras em Porto Alegre, 
estabelecendo-se padrões morfológicos que servirão de modelo para a identificação de diferentes espécies, contribuindo para a caracterização farmacognóstica das espécies de interesse como matéria-prima na indústria farmacêutica. Além disso, buscaram-se indícios de toxicidade, dados químicos e biológicos destas plantas.

\section{MATERIAL E MÉTODOS}

Foi realizada uma revisão bibliográfica de trabalhos efetuados em várias regiões do Estado do Rio Grande do Sul, referentes à utilização medicinal de espécies conhecidas popularmente como quebra-pedras. Os trabalhos revisados foram Garlet (2000), Marodin (2000), Possamai (2000), Ritter et al. (2002), Sebold (2003) e Vendruscolo (2004).

Foram adquiridas, mediante pagamento, no período de julho de 2005 a julho de 2006, 16 amostras em diferentes locais da cidade, de 14 comerciantes de plantas medicinais em bancas fixas ou em feiras-livre, nos bairros Centro e Bom Fim, locais de maior circulação de pessoas e, conseqüentemente, de comerciantes. As amostras encontravam-se como material fresco ou seco, em ramos inteiros ou rasurados.

O material adquirido foi analisado e identificado no laboratório de Taxonomia de Angiospermas, do Departamento de Botânica da Universidade Federal do Rio Grande do Sul, com auxílio da literatura específica e por comparação com material botânico depositado no Herbário ICN/UFRGS. Para a identificação das espécies de Phyllanthus, foi utilizado o trabalho de Machado (2003) e para as espécies de Euphorbia o trabalho de Allem (1975).

O sistema de classificação adotado para as famílias é o da APG II (2003).

Para cada espécie foi realizada uma busca de dados químicos e biológicos na literatura específica e em bases de dados, como Medline 2007 e Science Direct 2007.

\section{RESULTADOS E DISCUSSÃO}

Foram identificadas sete espécies pertencentes a cinco famílias:

\section{Cunila microcephala Benth. - Lamiaceae}

Nomes populares: poejinho (Sebold, 2003), poejo-cheiroso (Possamai, 2000), poejo (Possamai, 2000; Garlet, 2000; Marodin, 2000), poejo-miúdo (Marodin, 2000), poejo-graúdo (Vendruscolo, 2004). Usos: como tempero, para sinusite e bronquite (Possamai, 2000), tosse (Possamai, 2000; Sebold, 2003), gripe (Possamai, 2000; Garlet, 2000), calmante da tosse, problemas de peito, estômago e cólica de nenê (Garlet, 2000), para bronquite, dores de barriga, quando se está gripado e enjoado, para expectorar, em infecções e afecções dos pulmões, rinite e tosse (Vendruscolo, 2004). Caracteres morfológicos: folhas simples, opostas, pecioladas, glabras, com bordo serrado ou esparsamente dentado e tricomas glandulares (Fig. 1). Dados químicos e biológicos: o óleo essencial tem como principal componente o mentofurano e tujeno, além de limoneno, $\beta$-cariofileno e pulegona (Bordignon et al., 1997). O primeiro corresponde a uma hepatotoxina. Outros constituintes foram identificados no óleo essencial de folhas e flores, como limoneno, 1,8-cineol, linalol, mentofurano, L-copaeno, espatulenol, óxido de cariofileno, 2-pentadecanona, além de mentona e mentol. Foi relatada ação antidematogênica do extrato hidroalcoólico das partes aéreas da planta, em experimento com animais de laboratório. Oóleo essencial foi ineficaz em camundongos no teste de proteção a convulsões induzidas por "eletrochoque transcorneal máximo" (ETM). A ação "hepatotóxica" devido à presença do mentofurano (componente principal) no óleo essencial sugere precaução no uso desta espécie. A não comprovação da ação protetora a convulsões, não indica o uso da planta como calmante. Não há relatos que comprovem seu uso para cólicas, diarréias, gripe e tosse. É relatada atividade antidematogênica para a referida espécie (Marodin, 2000). A atividade antimicrobiana do óleo essencial foi efetiva contra espécies de Bacillus sp. Staphylococcus aureus, e outras bactérias específicas (Sandri et al., 2007).

\section{Desmodium incanum DC. - Fabaceae}

Nomes populares: pega-pega (Garlet, 2000; Sebold, 2003; Vendruscolo, 2004). Uso: em problemas de sangue, rins, bexiga e próstata; segura urina frouxa de criança (Garlet, 2000), para problemas nos rins (Sebold, 2003) e nos ovários (Vendruscolo, 2004). Caracteres morfológicos: folhas compostas pinado-trifolioladas, alternas, pecioladas, pilosas, com bordo inteiro e base aguda (Fig. 2). Dados químicos e biológicos: presença de flavonóides, taninos e saponinas (Bianchi et al., 1989). Não foi encontrada atividade tóxica no ensaio de toxicidade aguda em camundongos nas doses 250 e 500 $\mathrm{mg} / \mathrm{Kg}$ para extratos aquosos liofilizados (Bianchi et al., 1989) e mutagênica para o extrato aquoso no teste de Ames (Vargas et al., 1991).

\section{Euphorbia prostrata Aiton - Euphorbiaceae}

Nomes populares: quebra-pedras-roxo (Garlet, 2000), quebra-pedras-rasteiro (Sebold, 2003), quebrapedra (Vendruscolo, 2004). Uso: em infecção de garganta, inflamação e pedras nos rins (Garlet, 2000). Como diurético e para problemas nos rins (Sebold, 2003; Vendruscolo, 2004). Caracteres morfológicos: folhas simples, opostas, pecioladas, glabras, com bordo serrado ou esparsamente dentado e base assimétrica (Fig. 3). Dados químicos e biológicos: presença de 
ácido galico, corilagina, 1,2,3-tri-O-galloyl-D-glicose, geranina, telimagradina I, II, rugosina A, rugosina E, rugosina $\mathrm{D}$ e rugosina $\mathrm{G}$ (Chen et al., 1992), além de flavonóides (Singla \& Pathak, 1989; Pathak et al., 1991). Foram reportadas atividades antiinflamatórias (extrato acetato de etila da planta inteira; flavonóides: apigenina e luteolina) (Singla \& Pathak, 1989; Pathak et al., 1991) e efeito hipoglicêmico em coelhos normais (extrato metanólico do pó da planta) (Handa et al., 1989, Alarcon-Aguilara et al., 1998). As espécies de Euphorbia produzem um látex que em contato com a pele, pode causar lesões severas, como edemas, bolhas e pústulas. Em contato com os olhos, o látex pode causar conjuntivites ou até a cegueira temporária. $\mathrm{O}$ látex ou partes da planta, quando ingeridos, causam irritações na mucosa da boca, gastroenterite, diarréia, vômitos. A literatura médica registra casos de convulsões e lesões renais. O uso desta planta é fortemente desaconselhado no tratamento de qualquer sintoma ou doença, não devendo ser usada durante a gravidez (Vendruscolo, 2004).

\section{Euphorbia serpens Kunth - Euphorbiaceae}

Nomes populares: quebra-pedras (Garlet, 2000; Ritter et al., 2002; Vendruscolo, 2004), quebra-pedrabranco (Possamai, 2000), erva-pombinha (Vendruscolo, 2004). Uso: em cálculos renais, para bexiga, inflamação nos rins e como diurético (Garlet, 2000; Possamai, 2000; Ritter et al., 2002; Vendruscolo, 2004). Caracteres morfológicos: folhas simples, opostas, pecioladas, glabras, com bordo inteiro e base oblíqua (Fig. 4). Dados químicos e biológicos: presença de fenóis, flavonóides, cianidinas, taninos e saponinas nas folhas. (Paz et al., 1992). O látex contém resinas (Maffei, 1997).

\section{Heimia salicifolia Link - Lythraceae}

Nomes populares: erva da vida (Simões et al., 1986; Possamai, 2000; Sebold, 2003), erva-desanta-luzia, erva-das-vistas (Possamai, 2000), abreo-sol, vassourinha (Simões et al., 1986). Uso: para conjuntivite, lavar os olhos e fígado (Possamai, 2000). Internamente como diurética e purgante; externamente como cicatrizante; a planta toda é utilizada como repelente de insetos (Simões et al., 1986). As partes aéreas têm ampla reputação popular de atividades antisifilítica, sudorífera, antipirética, laxativa e diurética, e são relatados como úteis na preparação de banhos póspartos e de melhora na cura de ferimentos (Malone \& Rother, 1994). Caracteres morfológicos: folhas simples, opostas ou verticiladas, raro as superiores alternas, sésseis ou quase sésseis, glabras, com bordo inteiro e base cuneada (Fig. 5). Dados químicos e biológicos: presença de heterosídeos (Maffei, 1997). Foram isolados vários alcalóides: criogenina (vertina), litrina, sinicuichina, heimina, sinina, heimidina, nesodina, lifolina, litridina, dihidronesodina, 0-0-dimetilifolina (Ragonese et al., 1984). Os quatro alcalóides mais estudados são: vertina, liofilina, litrina e nesodina. Análises clínicas preliminares indicam que vertina e litrina aplicados oralmente aparentam cessar a atividade psicodisléptica; vertina tem potenciais tranquilizante, antiinflamatório e antiespasmódico; litrina têm potencial hidrodiurético (Malone \& Rother, 1994). Do extrato hidrolisado de folhas foi isolado ácido elágico, além de sitosterol e manitol em grandes quantidades. Apesar dos alcalóides possuirem atividade anticolinérgica, antiespasmódica e tranqüilizante, tais propriedades não explicam totalmente a utilização popular da planta como alucinógena, em determinadas regiões do México (Ragonese et al., 1984; Simões et al., 1986). O extrato aquoso das partes aéreas demonstrou intensa atividade antibacteriana contra Salmonella typhi (Pérez \& Anesini 1994).

\section{Phyllanthus niruri L. - Phyllanthaceae}

Nomes populares: erva-pombinha (Simões et al., 1986; Garlet, 2000), quebra-pedras-de-arvorezinha (Garlet, 2000), quebra-pedra (Simões et al., 1986; Ritter et al., 2002; Vendruscolo, 2004), quebra-pedra legítimo, quebra-pedra verdadeiro (Vendruscolo, 2004). Uso: para bexiga e rins (Garlet, 2000). Internamente as folhas são usadas como diuréticas, litolíticas, eupépticas, em afecções do fígado, icterícia, cólicas renais, moléstias da bexiga, retenção urinária e como auxiliar na eliminação de ácido úrico. As raízes são também utilizadas em afecções hepáticas com icterícia e os frutos, as sementes e as folhas em diabetes (Simões et al., 1986). Para dor nos rins, bexiga, urina trancada, pedra nos rins e como diurético (Ritter et al., 2002). Caracteres morfológicos: folhas simples, alternas, pecioladas, glabras, com bordo inteiro e base oblíqua (Fig. 6). Dados químicos e biológicos: Presença de flavonóides (quercetina I, astragalina, quercitrina e rutina XXI) (Santos, 1988), lignanas (filantina e hipofilantina) (Row et al., 1966), triterpenóides, alcalóides (filocrisina, filalvina) (Santos, 1988) e um alcalóide pirrolizidínico (Lorenzi \& Matos 2002), cineol, cimol, linalol, salicilato de metila, securimina, filantiolina, ácido salicílico (Martins, 1994). Foram isolados compostos fenólicos das raízes como ácido galico, (-)-epicatequina, $(+)$-galocatequina, (-)-epigalocatequina, (-)-epicatequina-3-O-galate e (-)-epigalocatequina- 3-O-galate. Das folhas e caule taninos hidrolisáveis como geranina, corilagina e galoilglicose (Ishimaru et al., 1992). Ainda a presença de princípios amargos, ácido ricinoléico e os extratos aquosos mostraram efeito hipoglicemiante (Simões et al., 1986). Apresenta ação antibacteriana (Staphylococcus), antiespasmódica e anticancerígena (Sousa et al., 1991). Ensaios mostraram que o extrato aquoso é ativo contra o vírus da hepatite B "in vitro" e "in vivo", por via intraperitonial, quando aplicado 

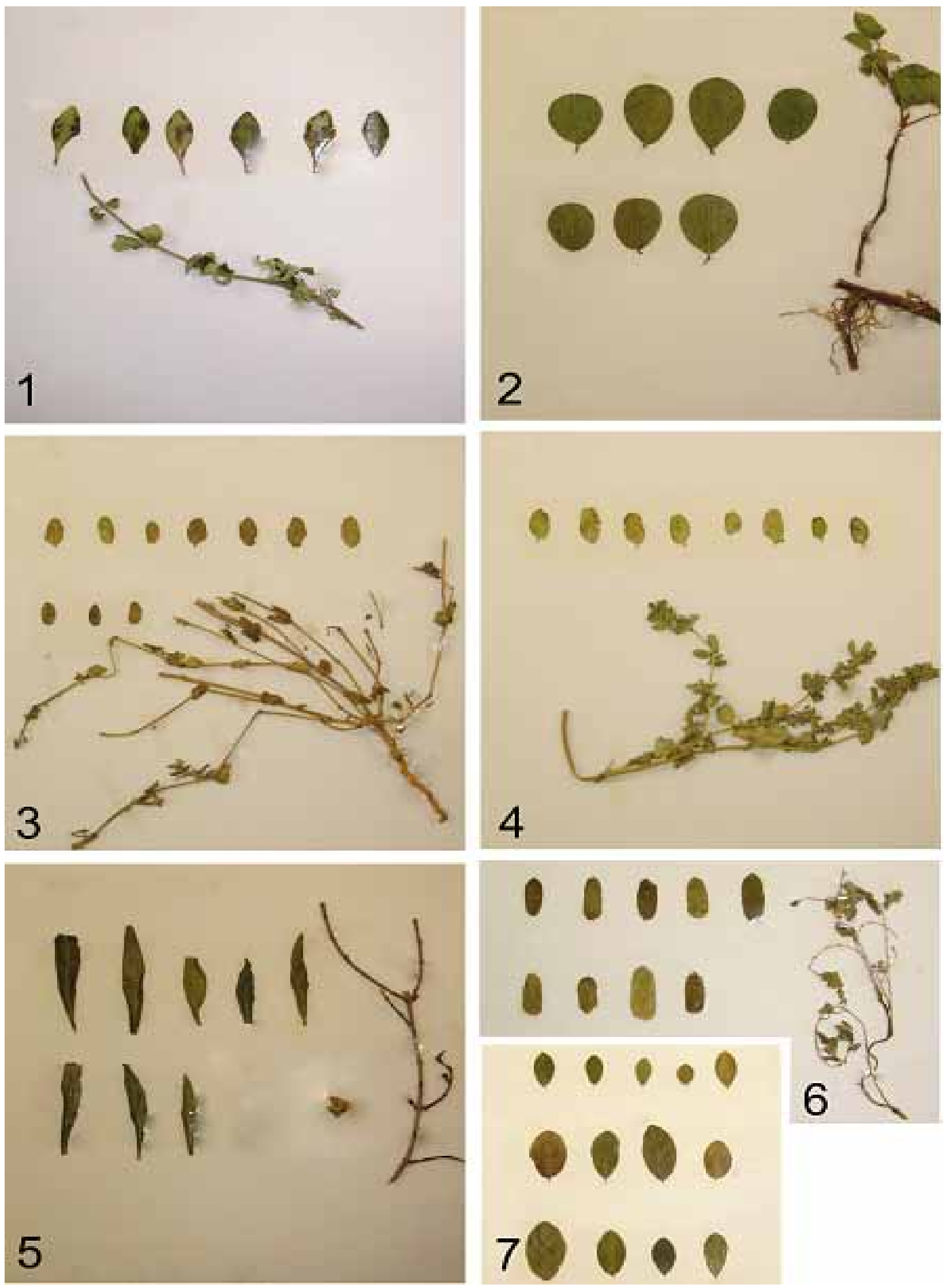

Figuras 1-7. Amostras de espécies comercializadas como quebra-pedras. 1. Cunila microcephala Benth. 2. Desmodium incanum DC. 3. Euphorbia prostrata Aiton 4. Euphorbia serpens Kunth 5. Heimia salicifolia Link 6. Phyllanthus niruri L. 7. Phyllanthus tenellus Roxb. 
em roedores infectados (Venkateswaran et al., 1987) além de ação antihepatotóxica (Syamasundar, 1985) ou hepatoprotetora e antioxidante (Harish \& Shivanandappa, 2006). Foi verificado através de análises clínicolaboratoriais que doses do infuso acima das comumente usadas não mostraram ação tóxica para o homem. Foram observados que extratos de P. niruri normalizaram os níveis altos de cálcio urinário diminuindo a formação dos cálculos em pacientes (Nishiura et al., 2004) sugerindo que deva interferir nos primeiros estágios de formação da pedra e pode representar uma alternativa no tratamento e/ou prevenção da urolitíase (Barros et al., 2003). Foi evidenciado uma elevação da filtração glomerular e da excreção urinária do ácido úrico. Os resultados obtidos podem justificar, pelo menos em parte, o uso medicinal da planta em certas afecções renais ou mesmo nos casos de insuficiência renal (Sousa et al., 1991). Estudos esclarecem que a utilização da planta promove um relaxamento dos ureteres que, aliado a uma ação analgésica (Santos et al., 1995), facilita a descida dos cálculos, geralmente sem dor nem sangramento, aumentando a filtração glomerular e a excreção de ácido úrico (justificando seu uso para pedra nos rins) (Lorenzi $\&$ Matos, 2002). O extrato aquoso inibe o virus humano da imunodeficiência tipo-1 trancriptase reversa (HIV-1RT) (Ogata et al., 1992). Ainda apresenta ação abortiva e purgativa em dosagens acima das normais (Martins et al., 1994).

\section{Phyllanthus tenellus Roxb. - Phyllanthaceae}

Nomes populares: quebra-pedras-dearvorezinha, erva-pombinha (Garlet, 2000); quebrapedra (Possamai, 2000; Marodin, 2000; Sebold, 2003; Vendruscolo, 2004), quebra-pedra-claro (Possamai, 2000), quebra-pedra falso, quebra-pedra graúdo (Vendruscolo, 2004). Uso: para bexiga, rins e como diurético (Garlet, 2000; Sebold, 2003), em casos de cálculo renal, cistite, corrimento, como diurética, em problemas dos rins e dos ovários, frio da bexiga, inflamações na bexiga e pedra nos rins (Vendruscolo, 2004). Caracteres morfológicos: folhas simples, opostas, pecioladas, glabras, com bordo inteiro e base atenuada (Fig. 7). Dados químicos e biológicos: presença de alcalóides, ácido gálico (Simões et al., 1986), flavonóides, terpenos, lignanas e fenóis (Calixto et al., 1997). Plantas do gênero Phyllanthus têm ações terapêuticas no tratamento de hepatite B e litíase e como antinociceptivo (Calixto et al., 1997). Os extratos fluídos e o decocto das partes aéreas deste vegetal não apresentaram atividade diurética, nem antiinflamatória nos ensaios em ratos (Simões et al., 1986). Atividade antinociceptiva foi relatada, em experimentos com ratos, a partir do extrato das folhas, caule e raízes. Testes em ratos com o extrato fluido não relataram o aumento da diurese, e nem sua atividade antiinflamatória, apresentando baixa toxicidade. Apresenta propriedades antibacterianas contra Aeromonas hydrophila, Bacillus subtilis, Pseudomonas aeruginosa e Staphylococcus aureus (Oliveira et al., 2007) e ação antiviral contra o vírus da hepatite B testado em patos (Shead et al., 1992).

Das espécies comercializadas como quebrapedras em Porto Alegre, as espécies de Phyllanthus niruri e Euphorbia serpens são as que apresentam maior semelhança morfológica quando comercializadas secas e destacadas dos ramos ou fragmentadas.

Nos trabalhos efetuados em outras regiões do Rio Grande do Sul, Desmodium incanum, Cunila microcephala e Heimia salicifolia são conhecidas por outros nomes populares. Provavelmente $D$. incanum e H. salicifolia estão sendo utilizadas atualmente para afecções renais e acabaram sendo denominadas também por quebra-pedras. Quanto à C. microcephala, provavelmente houve um engano por parte do comerciante, ao vender esta espécie como quebrapedras, já que é tradicionalmente utilizada para afecções do trato respiratório.

A grande maioria dos usos relatados pela população não encontra ainda respaldo nos estudos realizados até o momento.

As espécies do gênero Euphorbia contêm um látex extremamente cáustico, o qual pode causar lesões na pele (Santucci et al., 1985; Schmidt \& Evans, 1980), nos olhos (Scott \& Karp, 1996) e, quando ingerido, na mucosa gastrointestinal, pode levar a lesões renais (Schenkel et al., 2000).

Em várias amostras constatou-se a presença de material estranho, como pedaços de plástico, invertebrados, pedras e outras plantas. Além disso, várias se encontravam contaminadas por fungos, demonstrando a qualidade duvidosa destes materiais.

Estes dados demonstram que diferentes espécies conhecidas pelo mesmo nome popular de quebra-pedras são usadas indistintamente como medicinais, evidenciando a necessidade de estudos complementares e um controle de qualidade eficiente, para uma utilização eficaz e segura.

\section{AGRADECIMENTOS}

À FAPERGS pela bolsa de iniciação científica (processo número 05502482) concedida à primeira autora e ao botânico Ângelo Alberto Schneider pelo auxílio na editoração das figuras.

\section{REFERÊNCIAS}

Alarcon-Aguilara FJ, Roman-Ramos R, Perez-Gutierrez S, Aguilar-Contreras A, Contreras-Weber CC, FloresSaenz JL 1998. Study of the anti-hyperglycemic effect of plants used as antidiabetics. J Ethnopharmacol 61: 101-109.

Allem AC 1975. Estudo taxonômico do gênero Euphorbia L. (Euphorbiaceae) no Rio Grande do Sul. Porto Alegre, 
127p. Dissertação de Mestrado - Curso de PósGraduação em Botânica, Universidade Federal do Rio Grande do Sul.

APG II 2003. The Angiosperm Phylogeny Group. An update of the angiosperm phylogeny group classification for the orders and families of flowering plants: APG II. Bol J Linn Soc 141: 399-436.

Barros ME, Schor N, Boim MA 2003. Effects of an aqueous extract from Phyllantus niruri on calcium oxalate crystallization in vitro. Urol Res 30: 374-37 9.

Bianchi NR, Machado CA, Alice CB, Siqueira NC 1989. Riscos potenciais de algumas espécies utilizadas na medicina popular em afecções do aparelho respiratório. Rev Bras Farm 70: 68-70.

Bordignon SADL, Schenkel EP, Spitzer V 1997. The essential oil composition of Cunila microcephala and Cunila fasciculata. Phytochemistry 44: 1283-1286.

Calixto JB, Santos ARS, Paulino N, Cechinel Filho V, Yunes RA 1997. The plants of the genus Phyllanthus as a potential sourse of new drugs. Cienc Cult 49: 422-432.

Chen L, Chen R, Wei K 1992. Constituents of tannins from Euphorbia prostrata Aiton. Zhongguo Zhong Yao Za Zhi 17: 225-226.

Garlet TMB 2000. Levantamento das plantas medicinais utilizadas no municipio de Cruz Alta, RS, Brasil. Porto Alegre, 220p. Dissertação de Mestrado - Programa de Pós-Graduação em Botânica, Universidade Federal do Rio Grande do Sul.

Handa SS, Chawla AS, Maninder 1989. Hypoglycaemic plants - a review. Fitoterapia 60: 195-224.

Harish R, Shivanandappa T 2006. Antioxidant activity and hepatoprotective potential of Phyllanthus niruri. Food Chem 95: 180-185.

Ishimaru $\mathrm{K}$, Yoshimatsu $\mathrm{K}$, Yamakawa $\mathrm{T}$, Kamada $\mathrm{H}$, Shimomura K 1992. Phenolic constituents in tissue cultures of Phyllanthus niruri. Phytochemistry 31: 2015-2018.

Lorenzi H, Matos FJA 2002. Plantas medicinais no Brasil nativas e exóticas. Nova Odessa: Instituto Plantarum.

Machado CA 2003. Morfo-anatomia de espécies do gênero Phyllanthus L. (Euphorbiaceae). Porto Alegre, 113p. Tese de Doutorado - Programa de Pós-Graduação em Botânica, Universidade Federal do Rio Grande do Sul.

Maffei BRA 1997. Plantas usadas en medicina natural. Montevideo: Hemisferio Sur.

Malone MH, Rother A 1994. Heimia salicifolia: A phytochemical and phytopharmacologic review. J Ethnopharmacol 42: $135-159$

Marodin SM 2000. Plantas utilizadas como medicinais no município de Dom Pedro de Alcântara, Rio Grande do Sul. Porto Alegre, 413p. Dissertação de Mestrado - Curso de Pós-Graduação em Botânica, Universidade Federal do Rio Grande do Sul.

Martins ER, Castro DM, Castellani DC, Dias JE 1994. Plantas medicinais. Viçosa: UFV.

Medline 2007. Disponível em: <http://bases.bireme.br/>. Acesso em: 22 set. 2007.

Nishiura JL, Campos AH, Boim MA, Heilberg IP, Schor N 2004. Phyllanthus niruri normalizes elevated urinary calcium levels in calcium stone forming (CSF) patients. Urol Res 32: 362-366.

Ogata T, Higuchi H, Mochida S, Matsumoto H, Kato A, Endo T, Kaji A, Kaji H 1992. HIV-1 reverse transcriptase inhibitor from Phyllanthus niruri. AIDS Res Hum Retrov 8: 1937-1944.

Oliveira DF, Pereira AC, Figueiredo HCP, Carvalho DA, Silva G, Nune s AS, Alves DS, Carvalho HWP 2007. Antibacterial activity of plant extracts from Brazilian southeast region. Fitoterapia 78: 142-145.

Pathak D, Pathak K, Singla AK 1991. Flavonoids as medicinal agents - Recent advances. Fitoterapia 62: 371-389.

Paz EA, Bassagoda MJ, Ferreira F 1992. Uso racional de las plantas medicinales. Montevideo: Editorial Fin de Siglo.

Pérez C, Anesini C 1994. In vitro antibacterial activity of Argentine folk medicinal plants against Salmonella typhi. J Ethnopharmacol 44: 41-46.

Possamai RM 2000. Levantamento etnobotânico das plantas de uso medicinal em Mariana Pimentel, RS. Porto Alegre, 108p. Dissertação de Mestrado - Programa de Pós-Graduação em Botânica, Universidade Federal do Rio Grande do Sul.

Ragonese AE, Milano VA 1984. Vegetales y substancias tóxicas de la flora Argentina. Enciclopédia Argentina de Agricultura y Jardineria - tomo II, fascículo 8-2, 2 ed. Buenos Aires: ACME S.A.C.I.

Rates SMK 2001. Plants as source of drugs. Toxicon 39: 603613.

Ritter MR, Sobierajski GR, Schenkel EP, Mentz LA 2002. Plantas medicinais utilizadas no município de Ipê, Rio Grande do Sul. Rev Bras Farmacogn 12: 51-62.

Row LR, Srinivasulu C, Smith M, Rao GSRS 1966. Crystalline constituents of Euphorbiaceae-V: New lignans from Phyllanthus niruri Linn-The constitution of phyllanthin. Tetrahedron 22: 2899-2908.

Sandri IG, Zacaria J, Fracaro F, Delamare APL, Echeverrigaray S 2007. Antimicrobial activity of the essential oils of Brazilian species of the genus Cunila against foodborne pathogens and spoiling bacteria. Food Chem 103: 823-828.

Santos ARS, Filho VC, Yunes RA, Calixto JB 1995. Analysis of the mechanisms underlying the antinociceptive effect of the extracts of plants from the genus Phyllanthus. Gen Pharmacol 26: 1499-1506.

Santos CAM 1988. Plantas medicinais (herbarium, flora et scientia). 2.ed. São Paulo: Ícone, p.135.

Santucci B, Picardo M, Cristaudo A 1985. Contact dermatitis from Euphorbia pulcherrima. Contact Dermatitis 2: 285-286.

Schenkel EP, Zannin M, Mentz LA, Bordignon SAL, Irgang B 2000. Plantas tóxicas. In: Simões CMO, Schenkel, EP, Gosmann G, Mello JCP, Mentz LA, Petrovick PR 2000. Farmacognosia: da planta ao medicamento. Porto Alegre: Editora da Universidade/UFRGS e Florianópolis: Editora da UFSC, p.755-788.

Schmidt RJ, Evans FJ 1980. Skin irritants of the sun spurge (Euphorbia helioscopia L.). Contact Dermatitis 6: 204-210.

Science Direct 2007. Disponível em: $<$ http://www.sciencedirect. com>. Acesso em: 14 mai. 2007.

Scott IU, Karp CL 1996. Euphorbia sap keratopathy: four cases and a possible pathogenic mechanism. Brit $J$ Ophthalmol 80: 823-826.

Sebold DF 2003. Levantamento etnobotânico de plantas de uso medicinal no município de Campo Bom, Rio Grande do Sul, Brasil. Porto Alegre, 107p. Dissertação de Mestrado - Programa de Pós-Graduação em Botânica, Universidade Federal do Rio Grande do Sul.

Shead A, Vickery K, Pajkos A, Medhurst R, Freiman J, Dixon R, Cossart Y 1992. Effects of Phyllanthus plant extracts on duck hepatitis B virus in vitro and in vivo. Antiviral Res 18: 127-138.

Simões CMO, Mentz LA, Schenkel EP, Irgang BE, Stehmann JR 1986. Plantas da medicina popular no Rio Grande do Sul. 1.ed. Porto Alegre: UFRGS, p.60,72,73.

Singla AK, Pathak K 1989. Anti-inflammatory studies on 
Euphorbia prostrata. J Ethnopharmacol 27: 55-61.

Sousa MP, Matos MEO, Matos FJA, Machado MIL, Craveiro AA 1991. Constituintes químicos ativos de plantas medicinais brasileiras. Fortaleza: E.U.F.C.

Syamasundar KV 1985. Antihepatotoxic principles of Phyllanthus niruri herbs. J Ethnopharmacol 14: 4144.

Vargas VM, Guidobono RR, Henriques JA 1991. Genotoxicity of plant extracts. Mem Inst Oswaldo Cruz 86 (Suppl 2): $67-70$.

Vendruscolo GS 2004. Estudo etnobotânico das plantas utilizadas como medicinais por moradores do bairro Ponta Grossa, Porto Alegre, Rio Grande do Sul. Porto Alegre, 276p. Dissertação de Mestrado - Curso de Pós-Graduação em Botânica, Universidade Federal do Rio Grande do Sul.

Venkateswaran PS, Millman I, Blumberg BS 1987. Effects of an extract from Phyllanthus niruri on hepatitis B and Woodchuck hepatitis B viruses in vitro and in vivo studies. Proc Natl Acad Sci USA 84: 274-278.

Zuccolotto T, Apel M, Rates SMK 1999. Avaliação da qualidade de produtos fitoterápicos comercializados em Porto Alegre-RS. Rev Inst Adolfo Lutz 58: 25-31. 